\title{
Developmental changes in the acceptance of the five basic tastes in the first year of life
}

\author{
Camille Schwartz ${ }^{1,2,3}$, Sylvie Issanchou ${ }^{1,2,3 *}$ and Sophie Nicklaus ${ }^{1,2,3}$ \\ ${ }^{1}$ INRA, UMR 1129 FLAVIC, F-21000 Dijon, France \\ ${ }^{2}$ ENESAD, UMR 1129 FLAVIC, F-21000 Dijon, France \\ ${ }^{3}$ Université de Bourgogne, UMR 1129 FLAVIC, F-21000 Dijon, France \\ (Received 15 December 2008 - Revised 12 March 2009 - Accepted 25 April 2009 - First published online 9 June 2009)
}

Taste is a major determinant of children's food preferences, but its development is incompletely known. Thus, exploring infants' acceptance of basic tastes is necessary. The first objective was to evaluate the acceptance of tastes and their developmental changes over the first year. The second objective was to compare acceptance across tastes. The third objective was to evaluate global taste reactivity (within-subject variability of acceptance across tastes). Acceptance of sweet, salty, bitter, sour and umami tastes was assessed in three groups of forty-five 3-, 6- and 12-month-old infants using observations based on ingestion and liking scored by the experimenter. For each taste, four bottles were presented (water, tastant, tastant, water). Acceptance of each taste relative to water was defined using proportional variables based on ingestion or liking. Acceptance over the first year only evolved for sweet taste (marginal decrease) and salty taste (clear increase). At each age, sweet and salty tastes were the most preferred tastes. Reactions to umami were neutral. Sour and bitter tastes were the least accepted ones but rejected only when considering liking data. Ingestion and liking were complementary to assess taste acceptance. However, congruency between these measures rose during the first year. Moreover, with increasing age, reactions were more and more contrasted across tastes. Finally, during the first year, inter-individual variability increased for all tastes except salty taste. By enhancing knowledge of the development of taste acceptance the present study contributes to understand better food behaviour in infancy, the foundation of food behaviour in adulthood.

Infants: Taste: Preference: Reactivity

In the current context of an increasing prevalence of obesity, a majority of studies on food behaviour take only nutritional factors into account. However, since food behaviour is greatly guided by food preferences, and in particular by taste acceptance especially in the child population ${ }^{(1)}$, it is essential to take into account this psycho-affective aspect of eating to enhance our understanding of the development of food habits. For instance, vegetables often taste bitter (for example, cabbage, broccoli or chicory) or sour (for example, tomato), which could harm their consumption by children (for example, Capaldi \& Privitera ${ }^{(2)}$, Gibson et al. ${ }^{(3)}$, Havermans \& Jansen $^{(4)}$ and Nicklaus et al. ${ }^{(5,6)}$ ). Several studies suggest that food preferences and behaviour develop early in infancy ${ }^{(6,7)}$ and track further on until adulthood ${ }^{(8-11)}$. Thus, a better knowledge of the development of taste acceptance in infancy is necessary to contribute to a better understanding of food behaviour in infancy, the foundation of food behaviour in adulthood.

For several decades, the development of taste acceptance has aroused researchers' attention. These early studies raised the notion that at only a few hours after birth, human infants are able to discriminate tastes as demonstrated in studies relying on facial mimic or ingestion cues ${ }^{(12-14)}$ (for a review, see Ganchrow \& Mennella $\left.{ }^{(15)}\right)$. Thus, human newborns readily accept sweet taste but reject bitter taste ${ }^{(13)}$ despite the fact that taste acceptance might differ from one child to another ${ }^{(16)}$. Furthermore, human infants' taste abilities are very sharp: they can distinguish sweet solutions according to quantitative differences, as proved by their preference for sweeter solutions ${ }^{(14,17)}$ up to an optimal concentration ${ }^{(18)}$. They also prefer sugars with a higher sweetening power ${ }^{(19)}$. Developmental changes in the acceptance of salty taste have also been studied. At birth, reactions to an aqueous solution of salt are evasive and spread over neutral reactions to rejection $^{(16,18)}$. Beauchamp and colleagues demonstrated that the preference for salty water emerges at about the fourth month of life and is well marked in 6- to 24-month-old infants; however, this preference seems to decline around the ages of 31 to 60 months $^{(20,21)}$. Data concerning bitterness acceptance are somehow controversial. At birth, infants display a disgust face when exposed to quinine ${ }^{(13)}$, but ingestion studies failed to reveal a clear rejection of urea ${ }^{(12,22)}$. A decrease in the acceptance of urea presented in a mildly sweet solution appears between birth and the age of 6 months ${ }^{(22)}$, and

Abbreviations: IR, ingestion ratio; LR, liking ratio; MSG, monosodium glutamate; OPALINE, Observatory of Food Preferences in Infants and Children;

R-IR, global taste reactivity based on ingestion ratios; R-LR, global taste reactivity based on liking ratios.

* Corresponding author: Dr Sylvie Issanchou, fax +33 3806932 27, email issan@dijon.inra.fr 
similarly 2- to 24-month-old infants decrease their ingestion when urea is presented in a mildly sweet solution ${ }^{(23)}$. In the same way, sour taste is rejected at birth by human infants, who display negative mimics after presentation of a sour solution $^{(13,16)}$ and reduce their ingestion of a mildly sweet and sour solution compared with a sweet solution ${ }^{(12)}$. In older infants (aged 2-24 months), the addition of citric acid to a sweet solution also decreases its ingestion ${ }^{(23)}$. Recently, preferences for extremely sour fruit juices were shown in $23 \%$ of 15 - to 20 -month-old infants ${ }^{(24)}$ and in $35 \%$ of 5 - to 9-year-old children ${ }^{(25)}$. However, it remains unknown whether such heightened sour preferences are also present in some younger infants. Lastly, the few studies on the acceptance of umami taste showed that in newborns a glutamate-flavoured soup generates the same reactions as a sweet solution ${ }^{(26)}$. In older infants (aged 3-24 months), the acceptance of umami taste depends on the vehicle: added to water, monosodium glutamate (MSG) decreases its ingestion whereas added to a soup, it increases its ingestion ${ }^{(27)}$.

In summary, taste acceptance development is imperfectly described, in particular after the first 6 months of life, and for sour, bitter and umami tastes, despite the putative role of bitter and sour tastes in the rejection of foods bearing these tastes. Moreover, most previous taste acceptance studies were conducted with taste compounds presented at high concentrations, highly above the taste intensities generally encountered in foods. In addition, acceptance of the different tastes was not always studied using the same methodology (ingestion studies were based on volume consumed, whereas liking studies were based on facial mimics) or sometimes was studied in groups of subjects spanning a wide age range which makes the assessment of developmental changes in taste acceptance difficult. Finally, to our knowledge, no comparison of acceptance across tastes at a given age has been reported.

Therefore, the first objective of the present study was to evaluate the acceptance of the five basic tastes at several ages during the first year, thereby describing developmental changes in this period. The second objective was to compare acceptance across tastes at each studied age. The third objective was to evaluate within-subject variability of reactions across tastes, namely global taste reactivity, and to assess how it evolves during the first year. Thus, the acceptance of the five basic tastes (sweet, salty, bitter, sour and umami) was assessed in three groups of 3-, 6- and 12-month-old infants using observations based on ingestion and liking cues. Finally, for each objective mentioned above, conclusions from observations based on ingestion and liking cues were compared.

\section{Subjects and methods}

\section{General design}

Data were collected as a part of a programme aimed at understanding the formation of food preferences from birth up to the age of 2 years (Observatory of Food Preferences in Infants and Children; OPALINE). Participating mothers were recruited before the last trimester of pregnancy using leaflets and posters at doctors' and paediatricians' consulting rooms in maternity hospitals and clinics, pharmacies and day-care centres. Parents had to be of age to be included in the study.
The criterion for infant inclusion at birth was to be in good health. Among various measures, infant taste acceptance was assessed at the following corrected ages (i.e. ages considering the calculated delivery date, not the actual delivery date): 3,6 , 12 and 20 months. These ages were chosen in order to assess taste acceptance at crucial times in the establishment of food acceptance in infancy: at the age of 3 months food experience is generally restricted to milk; the sixth month often corresponds to the beginning of introduction of solid foods; at the age of 12 months the transition from baby food to table food is in progress; and the measure at 20 months old occurs before the development of food neophobia. The present study was conducted according to the guidelines laid down in the Declaration of Helsinki; the general OPALINE procedure and the taste acceptance procedure were approved by the local ethical committee (Comité de Protection de Personnes Est I Bourgogne). Written and informed consent was obtained from both parents for all infants.

\section{Subjects}

Here are reported data collected for infants who participated in the taste acceptance test at the age of 3 months ( $n$ 45), 6 months $(n 45)$ or 12 months $(n$ 45). Data for the 20-month-old infants are not reported here because the procedure used was slightly different.

\section{Stimuli}

For each taste, the solution was made from mineral water (Evian ${ }^{\circledR}$; Danone Group, Paris, France) and from food-grade or pharmacological-grade tastants (Jerafrance, Jeufosse, France). The molecules were chosen as they could be encountered by the infant in amniotic fluid during pregnancy or in his/her diet either in breast or formula milk: lactose for sweet taste, sodium chloride for salty taste, urea for bitter taste and MSG for umami taste, or in solid foods later in his/her diet: citric acid for sour taste. The concentrations were chosen to be above adult detection thresholds, since infant detection thresholds might be in the same range as those of adults ${ }^{(28)}$. They were also selected to generate moderate intensities, to avoid stereotypical reactions observed in some studies using high concentrations ${ }^{(13)}$, in order to favour observations of varied reactions for a given tastant. Moreover, the concentrations were chosen in order to generate intensities close to the one produced by a $0.20 \mathrm{M}$ concentration of lactose which corresponds to its concentration in human milk $^{(29)}$. The concentrations were $0.085 \mathrm{M}$ for sodium chloride, $0 \cdot 18 \mathrm{M}$ for urea, $0.006 \mathrm{M}$ for citric acid and 0.009 $\mathrm{M}$ for MSG. Sensory tests conducted with an adult panel confirmed that, on average, these supra-threshold concentrations generated perceptions of moderate intensities, and that solutions prepared every 2 or $3 \mathrm{~d}$ did not taste different. So the solutions were prepared every second (for citric acid and MSG) or third day (for lactose, sodium chloride and urea) and were kept refrigerated at $+4{ }^{\circ} \mathrm{C}$. For infant testing, solutions were presented at room temperature in commercially available $150 \mathrm{ml}$ plastic bottles fitted with the nipple that the infant was used to (same shape and same material), according to the information provided by the mother before the test sessions. 


\section{Procedure}

Infants were tested individually in a room designed for infant testing at the Centre Européen des Sciences du Goût (Dijon, France) or at the FLAVIC (FLAvour VIsion Consumer Behaviour) joint research unit (Dijon, France) in the presence of one of their parents, usually the mother. At each age, infants participated in two videotaped sessions at approximately the same time of the day, chosen according to the parent's availability and to the infant's rhythm. The day interval between both sessions was as limited as possible (at 3 months old: 3 (SD 3) d; at 6 months old: 5 (SD 4) d; at 12 months old: 4 (SD 4) d).

The procedure was adapted from previously proposed methods ${ }^{(20,30)}$. It consisted of brief sequential presentations of bottles containing either a tastant solution or water. The procedure was identical for all three age groups, making it possible to compare results across age groups, but was suitable to each age-related developmental stage (i.e. at the ages of 3 and 6 months infants sat in a bouncer and at the age of 12 months in a high chair). The parent was asked to give water or milk to his/her infant $1 \mathrm{~h}$ before the test session and then not to give food or drink before coming. This helped to limit variability (between and within infants) in thirst and hunger state and its potential impact on acceptance of the different tastants. On the test day, the parent was asked when the infant's last meal had taken place to check compliance with study instructions. For each taste, a fixed sequence of four bottles (water - tastant - tastant - water) was presented to the infant by the experimenter to limit any parental influence. Within a sequence, each bottle was presented for $45 \mathrm{~s}$ with a $15 \mathrm{~s}$ pause between bottles. If the bottle was not readily accepted by the infant, it was offered several times for $45 \mathrm{~s}$; its presentation stopped only if the infant rejected it strongly, when he/she cried vigorously for example. Between each sequence of four bottles, a pause of at least 1 min was allowed. For each age, the five sequences corresponding to the five tastes were presented in a double-blind balanced order, over the two sessions.

Volumes presented were limited to $30 \mathrm{ml}$ for 3 - and 6-month-old infants and to $50 \mathrm{ml}$ for 12-month-old infants, except for urea, the volume for which was limited to $15 \mathrm{ml}$ whatever the age. To determine ingestion, bottles were weighed before and after consumption to the nearest $0.1 \mathrm{~g}$ (Sartorius U3600S; Sartorius AG, Göttingen, Germany). For each bottle, the experimenter who was blind to the tastants evaluated the infant's liking of the stimulus, using a fivepoint scale ranging from $1=$ 'strong rejection', $2=$ 'slight rejection', $3=$ 'neutral reaction', $4=$ 'slight acceptance' to $5=$ 'strong acceptance'. The experimenters were told to take into consideration the infant's global behaviour including facial mimics. For example, if the infant spitted out, frowned, pushed the bottle away and stopped to drink, the experimenter marked $1=$ 'strong rejection' whereas if the infant smiled, displayed a relaxed face, caught the bottle and drank, the experimenter marked $5=$ 'strong acceptance'.

Four carefully trained experimenters carried out the experiment. To begin the presentation of a bottle, the nipple was lightly rubbed against the lips. If the infant refused to drink he/she was offered a toy to increase the compliance with the testing protocol. When necessary the parent was seated close to his/her infant or the infant was seated on his/her parent's lap.

\section{Variables}

For each taste, analysis was restricted to infants who consumed at least $1.0 \mathrm{~g}$ from two bottles over a sequence. Acceptance was evaluated using two variables: an ingestion ratio (IR) based on volume consumed and a liking ratio (LR) based on the experimenter's judgment of the infant's liking, estimated through the infant's global behaviour.

Acceptance variables. For each tastant, the IR was defined as the ingestion volume of this tastant relative to the sum of ingestion volumes of this tastant and of water (i.e. $\mathrm{IR}_{\text {saltytaste }}=$ (total ingested volume of salt solution/(total ingested volume of salt solution + total ingested volume of water))). A ratio of 0.5 indicates equal consumption of water and of the tastant solution, i.e. indifference to the tastant. In contrast, a ratio higher than 0.5 indicates a preference for the tastant over water and a ratio lower than 0.5 indicates a rejection of the tastant over water. Using a proportional variable makes comparisons across ages and across individuals possible, eliminating individual- and age-related differences in sucking behaviour. Likewise, an LR was calculated for the scores of infant's liking evaluated by the experimenter. The IR and the LR spanned from 0 to 1 .

Reactivity variables. To assess within-subject variability of reactions, namely the infant's global taste reactivity across the five tastes, two variables derived respectively from IR and LR were defined taking into account data from all assessed tastes. The taste reactivity R-IR was defined as the standard deviation of the five IR. Likewise, the taste reactivity R-LR was defined as the standard deviation of the five LR. The analysis was restricted to infants for whom ratios were assessed at least for three tastes out of five. The higher these variables, the more the infant reacted differently to the different tastes.

\section{Statistical analyses}

Statistical analyses were carried out using SAS version 9.1 (SAS Institute Inc., Cary, NC, USA). All results are expressed as mean values and standard deviations. Student's $t$ tests were carried out to determine whether there were differences in birth weight and birth length between the three groups of infants. In the same way, $\chi^{2}$ tests were performed to assess whether groups were different concerning sex and milk feeding mode at birth.

For all studied ages, preliminary ANOVA were performed to assess whether there were differences among experimenters, among sessions or according to stimuli order in outcome variables (IR and LR). As the analysis did not reveal any significant influence of these factors on outcome variables, data were therefore combined across experimenters, sessions and stimuli order for further analyses. For each age, we assessed whether sex had an influence on infants' acceptance of the taste stimuli. No effect and no interaction between sex and tastant were observed whatever the outcome variables. For each age, we assessed whether the weight-for-length $Z$-score calculated according to the WHO Child Growth Standards was correlated to infants' taste acceptance variables. No effect was observed whatever the outcome variables. 
For each taste and each age, Student's $t$ tests were used to assess whether the acceptance variable (i.e. IR, LR) was different from 0.5 (i.e. whether there was a significant rejection or preference of the tastant over water). Furthermore, for each taste and each acceptance variable, ANOVA were carried out to assess the effect of age according to the following model: IR $($ or $\mathrm{LR})=$ age + error. Moreover, for each age, ANOVA were performed to assess whether acceptance varied across tastes (model IR (or LR) $=$ subject + taste + error). Additionally, ANOVA were performed to assess whether infants' global taste reactivity evolved during the first year of life (model R-IR (or R-LR) = age + error). This last analysis was restricted to infants for whom at least three IR or LR out of five were available, and the corresponding ANOVA were weighted with the number of taste acceptance variables available for each infant. The SAS general linear model (GLM) procedure was used for all ANOVA. Significance was examined on the basis of type III sum of squares tables. When the ANOVA revealed a significant effect $(P<0.05)$ or a marginal effect $(P<0 \cdot 10)$, least square means were calculated and $t$ tests were carried out to compare them. Moreover, for each taste and each acceptance variable (IR and LR), a Levene's test was performed in order to explore whether the variability increased over the first year of life. Finally, to assess whether IR and LR, and R-IR and R-LR were correlated, Kendall correlations were calculated.

\section{Results}

\section{Subjects' characteristics}

The characteristics of the mother-infant dyads are listed in Table 1. Among the forty-five infants who participated at each age, two infants at 3 months old, three at 6 months old and two at 12 months old did not comply with the experimental procedure. For some tastes, not all individual data could be taken into account because some infants did not complete the concerned sequence or did not meet the ingestion criteria (see Fig. 1 for the number of infants per age and acceptance variable).

There were no significant differences between the three groups in birth weight or in birth length. The $\chi^{2}$ tests did not reveal significant differences between groups concerning sex and milk feeding mode at birth. The three groups were very similar in terms of mothers' characteristics.

Infants' acceptance of each taste at the ages of 3, 6 and 12 months

The results are summarised in Fig. 1 where evolution of acceptance of each taste across ages for each acceptance variable can be read in rows.

Sweet taste acceptance. On average, IR was 0.56 (SD 0.11) at the age of 3 months, 0.60 (SD 0.13) at the age of 6 months and 0.55 (SD 0.17 ) at the age of 12 months.

Table 1. Infants' and mothers' characteristics

(Mean values and standard deviations or numbers and percentages)

\begin{tabular}{|c|c|c|c|c|c|c|}
\hline \multirow[t]{2}{*}{ Age group... } & \multicolumn{2}{|c|}{3 months old } & \multicolumn{2}{|c|}{6 months old } & \multicolumn{2}{|c|}{12 months old } \\
\hline & Mean & SD & Mean & SD & Mean & SD \\
\hline \multicolumn{7}{|l|}{ Infants' characteristics } \\
\hline $\mathrm{Age}^{\star}(\mathrm{d})$ & 98 & 12 & 190 & 11 & 371 & 12 \\
\hline Corrected age* $(\mathrm{d})$ & 92 & 9 & 183 & 8 & 362 & 8 \\
\hline \multicolumn{7}{|l|}{$\operatorname{Sex}(n)$} \\
\hline Girl & \multirow{2}{*}{\multicolumn{2}{|c|}{$\begin{array}{l}22 \\
23\end{array}$}} & \multirow{2}{*}{\multicolumn{2}{|c|}{$\begin{array}{l}23 \\
22\end{array}$}} & \multicolumn{2}{|c|}{25} \\
\hline Boy & & & & & \multicolumn{2}{|c|}{20} \\
\hline Birth weight (kg) & $3 \cdot 32$ & 0.46 & 3.38 & 0.54 & $3 \cdot 28$ & 0.49 \\
\hline Birth length (cm) & 49.7 & $2 \cdot 1$ & 50.2 & $2 \cdot 2$ & 49.9 & $2 \cdot 1$ \\
\hline Weight at test time $(\mathrm{kg})$ & 5.85 & 0.73 & 7.38 & 0.69 & 9.64 & 1.03 \\
\hline Length at test time $(\mathrm{cm})$ & $59 \cdot 7$ & $3 \cdot 2$ & $66 \cdot 8$ & $2 \cdot 4$ & 75.4 & $2 \cdot 3$ \\
\hline \multicolumn{7}{|l|}{$\begin{array}{l}\text { Milk feeding mode at birth } \\
\text { Exclusively breast-fed }\end{array}$} \\
\hline $\begin{array}{l}\text { Exciusively diedst-ied } \\
n\end{array}$ & \multicolumn{2}{|c|}{35} & \multicolumn{2}{|c|}{41} & \multicolumn{2}{|c|}{33} \\
\hline$\%$ & \multicolumn{2}{|c|}{78} & \multicolumn{2}{|c|}{91} & \multicolumn{2}{|c|}{73} \\
\hline \multicolumn{7}{|l|}{ Breast- and formula-fed } \\
\hline$n$ & \multicolumn{2}{|c|}{5} & \multicolumn{2}{|c|}{0} & \multicolumn{2}{|c|}{7} \\
\hline$\%$ & \multirow{2}{*}{\multicolumn{2}{|c|}{11}} & \multicolumn{2}{|c|}{0} & \multicolumn{2}{|c|}{16} \\
\hline \multicolumn{6}{|l|}{ Exclusively formula-fed } & \\
\hline$n$ & \multicolumn{2}{|c|}{5} & \multicolumn{2}{|c|}{4} & \multicolumn{2}{|c|}{5} \\
\hline$\%$ & \multirow{2}{*}{\multicolumn{2}{|c|}{11}} & & & & \\
\hline Infants fed solid foods $†$ & & & & & & \\
\hline$n$ & & & & & & \\
\hline$\%$ & & & & & & \\
\hline Mothers' characteristics & & & & & & \\
\hline Age (years) & 31 & 4 & 31 & 4 & 31 & 5 \\
\hline BMI $\left(\mathrm{kg} / \mathrm{m}^{2}\right)$ & $22 \cdot 4$ & 4 & $22 \cdot 9$ & 3 & $22 \cdot 4$ & 3 \\
\hline Caesarean section (\%) & & & & & & \\
\hline Multiparous (\%) & & & & & & \\
\hline
\end{tabular}

${ }^{*}$ At the first session.

† At test time. 

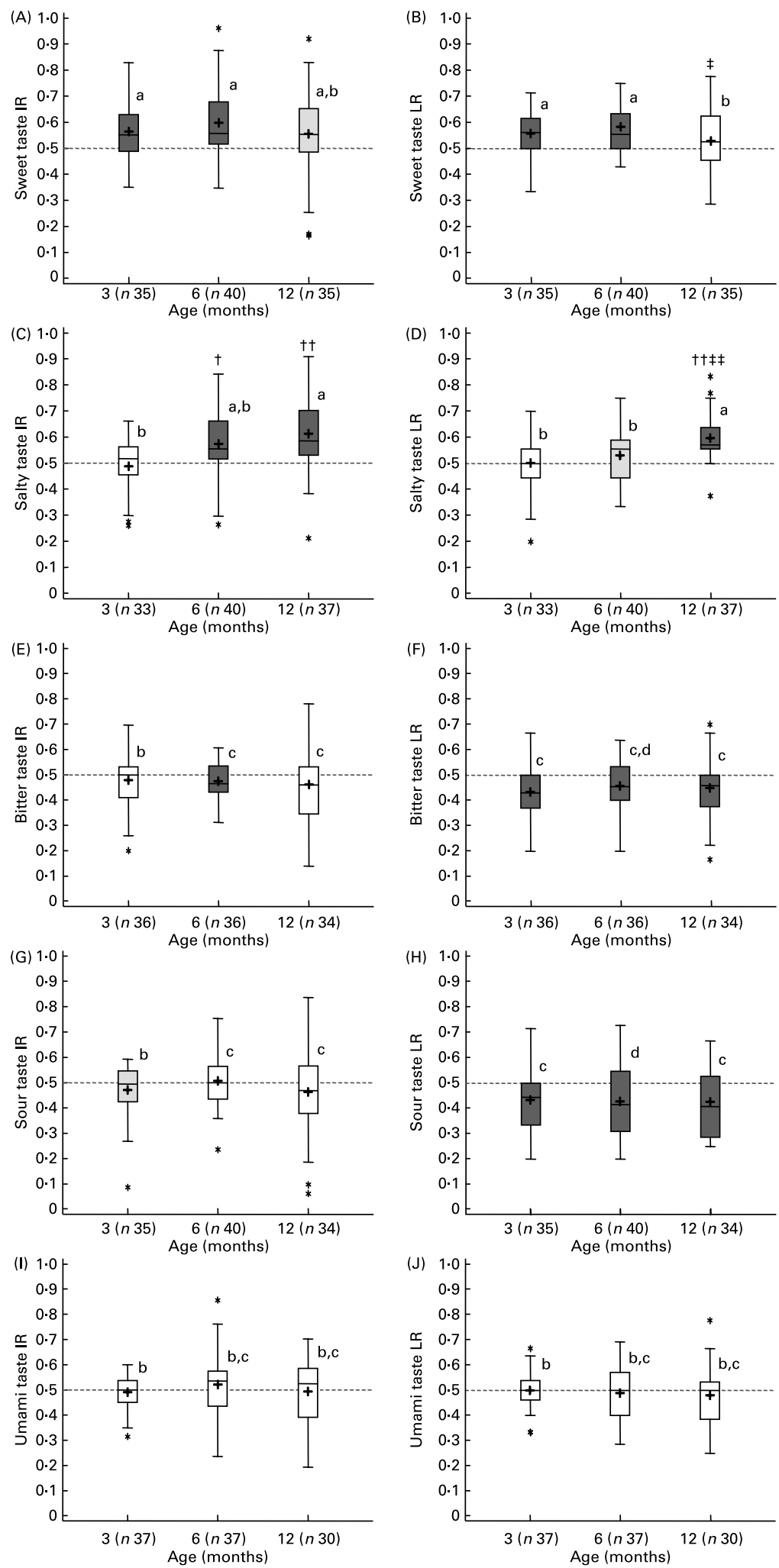

Fig. 1. Box plots of ingestion ratios (IR; $A, C, E, G, I)$ and liking ratios (LR; $B, D, F, H, J)$ for each taste (sweet (A, B), salty (C, D), bitter (E, F), sour (G, H) and umami (I, J) tastes) and for each studied age ( 3,6 and 12 months old). For each box plot, the bottom and the top of the box are the 25th and 75th percentiles and the line within the box is the median; the + sign is the mean. The whiskers extend from the box as far as the data extend, to a distance of at most $1.5 \times$ interquartile range. Any values more extreme than this are marked by $a^{*}$. Mean value was significantly different from $0.5(---)$ : $\square, P<0.10$ (marginal); $\square, P<0.05$ ( $t$ tests). Mean value was significantly different from that at 3 months: $\dagger P=0.008$, $\dagger+P=0.0001$ (LSMEANS and $t$ tests). Mean value was significantly different

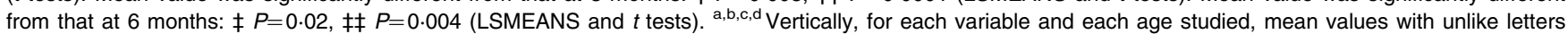
were significantly different $(P<0.05)$ (ANOVA; LSMEANS and $t$ tests). 
Infants significantly preferred sweet taste over water at the ages of $3\left(t_{34}=3.32 ; P=0.002\right)$ and 6 months $\left(t_{39}=4.82\right.$; $P<0.0001)$. This preference was marginally significant at the age of 12 months $\left(t_{34}=1.93 ; P=0.06\right)$. Examination of LR reveals similar results. On average, LR was 0.56 (SD 0.08) at the age of 3 months, 0.58 (SD 0.09) at the age of 6 months and 0.53 (SD 0.12 ) at the age of 12 months. Infants significantly preferred sweet taste over water at the ages of $3\left(t_{34}=4.29 ; P=0.0001\right)$ and 6 months $\left(t_{39}=5.65\right.$; $P<0.0001)$, but were indifferent to sweet taste at the age of 12 months $\left(t_{34}=1 \cdot 33 ; P=0 \cdot 19\right)$. For IR, the ANOVA showed no significant effect of age $(F(2,107)=1 \cdot 10$; $P=0.34)$; but for LR the ANOVA revealed a marginal effect of age $(F(2,107)=2.78 ; P=0.07)$ : the preference for sweet taste over water decreased between the ages of 6 and 12 months. Both for IR and LR, data revealed large inter-individual differences and some infants even rejected sweet taste over water. This phenomenon was observed for 31, 20 and $29 \%$ of the infants according to IR and for 9, 8 and $26 \%$ according to LR at the age of 3,6 and 12 months respectively.

Salty taste acceptance. On average, IR was 0.49 (SD 0.10) at the age of 3 months, 0.57 (SD 0.13) at the age of 6 months and 0.61 (SD 0.15) at the age of 12 months. According to IR, infants were indifferent to salty taste at 3 months old ( $\left.t_{32}=-0.59 ; P=0.56\right)$, but they significantly preferred salty taste over water at the ages of $6\left(t_{39}=3.50 ; P=0.001\right)$ and 12 months $\left(t_{36}=4.62 ; P<0.0001\right)$. Results based on LR were almost the same. On average, LR was 0.50 (SD 0.11), $0.53(\mathrm{SD} 0.10)$ and $0.59(\mathrm{SD} 0.09)$ respectively at the ages of 3,6 and 12 months. Infants were indifferent to salty taste over water at the age of 3 months $\left(t_{32}=0.03 ; P=0.98\right)$, marginally preferred salty taste over water at the age of 6 months $\left(t_{39}=1.78 ; P=0.08\right)$ and significantly preferred salty taste over water at the age of 12 months $\left(t_{36}=6.55 ; P<0.0001\right)$. The acceptance of salty taste over water significantly rose during the first year both for $\operatorname{IR}(F(2,107)=8.22$; $P=0.0005)$ and $\operatorname{LR}(F(2,107)=8 \cdot 38 ; P=0.0004)$. It evolved from indifference at 3 months old to a marked preference at 12 months old. Although reactions to salty taste were spread from indifference (3-month-old infants) to preference (6- and 12-month-old infants), some infants rejected salty taste over water. At 3, 6 and 12 months old respectively, this phenomenon concerned 46, 23 and $16 \%$ of the infants according to IR and 37, 28 and $3 \%$ according to LR.

Bitter taste acceptance. On average, IR was 0.48 (SD 0.11) at the age of 3 months, 0.47 (SD 0.08) at the age of 6 months and 0.46 (SD 0.16) at the age of 12 months. Infants were indifferent to bitter taste compared with water at the ages of 3 months $\left(t_{35}=-1 \cdot 28 ; P=0 \cdot 21\right)$ and 12 months $\left(t_{33}=-1 \cdot 38\right.$; $P=0 \cdot 18)$ but they rejected bitter taste over water at the age of 6 months $\left(t_{35}=-2.08 ; P=0.04\right)$. Conclusions from LR were slightly different; on average LR was 0.43 (SD 0.10), 0.46 (SD 0.10$)$ and $0.45(\mathrm{SD} 0 \cdot 12)$ at the ages of 3,6 and 12 months, respectively. According to LR, at all ages, infants rejected bitter taste over water (respectively at 3, 6 and 12 months old: $t_{35}=-3.88 \quad(P=0.0004) ; t_{35}=-2.56$ $\left.(P=0.02) ; t_{33}=-2.49(P=0.02)\right)$. The acceptance of bitter taste over water did not evolve between the ages of 3 and 12 months (for IR: $F(2,103)=0.15(P=0.86)$; for LR: $F(2,103)=0.47 \quad(P=0.63))$. Concerning inter-individual differences, some infants preferred bitter taste over water.
This was the case for 47, 33 and $38 \%$ of the infants according to IR and for 14,31 and $21 \%$ according to LR at the ages of 3, 6 and 12 months respectively.

Sour taste acceptance. On average, IR was 0.47 (SD 0.10) at the age of 3 months, 0.51 (SD $0 \cdot 11$ ) at the age of 6 months and 0.47 (SD 0.16) at the age of 12 months. Infants marginally rejected sour taste over water at the age of 3 months $\left(t_{34}=-1.78 ; P=0.08\right)$ but were indifferent to sour taste compared with water at the ages of $6\left(t_{39}=0.31 ; P=0.76\right)$ and 12 months $\left(t_{33}=-1 \cdot 26 ; P=0.22\right)$. Conclusions from LR are different: on average LR was 0.43 (SD 0.12) at the age of 3 months, 0.43 (SD 0.14 ) at the age of 6 months and 0.43 (SD 0.13) at the age of 12 months. LR showed that infants rejected sour taste at all ages (respectively at the ages of 3,6 and 12 months: $t_{34}=-3.35 \quad(P=0.002) ; \quad t_{39}=-3.42$ $\left.(P=0.002) ; \quad t_{33}=-3.29 \quad(P=0.002)\right)$. The acceptance of sour taste over water did not change between the ages of 3 and 12 months (for IR: $F(2,106)=1.16(P=0.32)$; for LR: $F(2,106)=0.03(P=0.97))$. As for bitter taste, some infants showed a preference for sour taste over water: 43,48 and $44 \%$ of the infants for IR and 23, 33 and $26 \%$ for LR respectively at the ages of 3,6 and 12 months.

Umami taste acceptance. On average, IR was 0.49 (SD 0.08) at the age of 3 months, 0.52 (SD 0.13) at the age of 6 months and 0.49 (SD $0 \cdot 14$ ) at the age of 12 months. Thus, infants were indifferent to umami taste compared with water at all ages (respectively at the ages of 3, 6 and 12 months: $t_{36}=-0.98 \quad(P=0.34) ; t_{36}=0.83 \quad(P=0.41)$; $\left.t_{29}=-0.25 \quad(P=0.80)\right)$. Similar results were obtained for LR, being $0.50(\mathrm{SD} 0.08$ ) at the age of 3 months, 0.49 (SD 0.10$)$ at the age of 6 months and 0.48 (SD 0.13) at the age of 12 months. Infants were indifferent to umami taste (respectively at the ages of 3,6 and 12 months: $t_{36}=0.12$ $\left.(P=0.90) ; t_{36}=-0.67(P=0.51) ; t_{29}=-0.93 \quad(P=0.36)\right)$. The acceptance of umami taste over water did not evolve between the ages of 3 and 12 months (for IR: $F(2,101)=0.69 \quad(P=0.50)$; for $\mathrm{LR}: \quad F(2,101)=0.44$ $(P=0 \cdot 65))$. Large inter-individual differences existed between infants in the acceptance of umami taste.

It must be pointed out that the inter-individual variability in acceptance was large within taste, and that this variability increased with age for some tastes and some variables (see Levene's test on IR and/or LR). This increased variability with age was particularly significant for bitter taste for IR $(F(2,103)=7 \cdot 87 ; P=0.0007)$. For umami, it was observed both for IR $(F(2,101)=4.17 ; P=0.02)$ and LR $(F(2,101)=4.07 ; P=0.02)$. It increased slightly for sweet taste for $\operatorname{LR}(F(2,107)=3.43 ; P=0.04)$ and tended to increase for sour taste for IR $(F(2,106)=2.65 ; P=0.08)$. Thus, over the first year, reactions to tastes were more and more contrasted across infants for all the tastes except for salty taste.

Taste acceptance across the five tastes at the ages of 3, 6 and 12 months

The results are presented in Fig. 1 where comparisons across tastes for each studied age can be read in columns.

At the age of 3 months, the ANOVA conducted to analyse the taste effect on IR showed that infants significantly accepted sweet taste over water more than salty, bitter, sour and umami tastes $(F(4,129)=6.03 ; \quad P=0.0002)$. 
Results based on LR were more contrasted: sweet taste was the most accepted taste, whereas bitter and sour tastes were the less accepted tastes $(F(4,129)=11.48 ; P<0.0001)$.

At the age of 6 months, according to IR, the acceptance of sweet and salty tastes was significantly higher than the acceptance of bitter and sour tastes. Umami taste was as accepted as salty, sour and bitter tastes $(F(4,147)=7 \cdot 22 ; P<0.0001)$. The results were similar for LR except that sweet taste was more accepted than salty taste and that umami taste was more accepted than sour taste $(F(4,147)=14.89$; $P<0.0001)$. As observed at the age of 3 months, LR provided more contrasted results.

At 12 months old, according to IR, salty taste was as accepted as sweet taste but more accepted than umami, sour and bitter tastes. Moreover, sweet taste was as accepted as umami taste but more accepted than sour and bitter tastes. Finally, bitter, sour and umami tastes were as much accepted as each other $(F(4,123)=6.89 ; P<0.0001)$. Conclusions from LR were rather more contrasted $(F(4,123)=13 \cdot 50$; $P<0.0001)$ : salty taste was the most liked taste, sweet taste was as accepted as umami taste, and bitter and sour tastes were the least accepted tastes.

\section{Global taste reactivity at the ages of 3, 6 and 12 months}

Concerning R-IR, the ANOVA showed a significant effect of age: R-IR significantly increased over the first year $(F(2,110)=5.70 ; \quad P=0.004) \quad($ Fig. 2$)$. R-IR marginally increased between the ages of 3 and 6 months and then between the ages of 6 and 12 months; R-IR significantly increased between the ages of 3 and 12 months. R-LR significantly increased between the ages of 3 and 12 months $(F(2,110)=3.06 ; P=0 \cdot 05)$ but to a lesser extent than R-IR. Either for ingestion or for liking, infants displayed an increasing reactivity across different taste stimulations over the first year.

\section{Congruency between ingestion and liking}

Taste acceptance: correlations between ingestion ratio and liking ratio. Table 2 shows that IR and LR were more correlated at the age of 6 months than at the age of 3 months and more correlated at the age of 12 months than at the age of 6 months with few exceptions for each taste. When infants grew up, ingestion and liking became more congruent.

Global taste reactivity: correlations between global taste reactivity based on ingestion ratios and global taste reactivity based on liking ratios. Concerning taste reactivity, R-IR and R-LR were not correlated at the age of 3 months $\left(\tau_{(35)}=0 \cdot 13\right.$; $P=0.26)$; they were marginally correlated at the age of 6 months $\left(\tau_{(39)}=0 \cdot 19 ; P=0 \cdot 08\right)$ and significantly correlated at the age of 12 months $\left(\tau_{(33)}=0.38 ; P=0.001\right)$. The congruency between both indicators of global taste reactivity became higher over the first year.

\section{Discussion}

The present study highlights developmental changes during the first year of life in the acceptance of sweet and salty tastes but not in the acceptance of bitter, sour and umami tastes. In addition, the present study is the first to assess acceptance of the basic tastes in the same group of infants at a given age, thus giving the opportunity to compare acceptance across the five basic tastes at crucial times in the formation of food behaviour. On average, sweet and salty tastes were the most accepted tastes whereas sour and bitter tastes were the least accepted ones. However, bitter and sour tastes were not systematically rejected in the sense that sour and bitter solutions were on average hardly ever significantly less consumed than water. Moreover, the present study compared conclusions from two different observational measures: ingestion and liking. Both measures did not systematically lead to the same conclusions; however, the congruency between conclusions drawn from both measures rose over the first year. These measures were therefore complementary to assess taste acceptance in infants, and helped to clarify previous conflicting results obtained with only one measure. Finally, the findings of the present study point out the fact that acceptance varied not only according to tastes but also according to infants. Therefore, the acceptance of the basic tastes does not seem to be as stereotyped as it has often been described up to now.
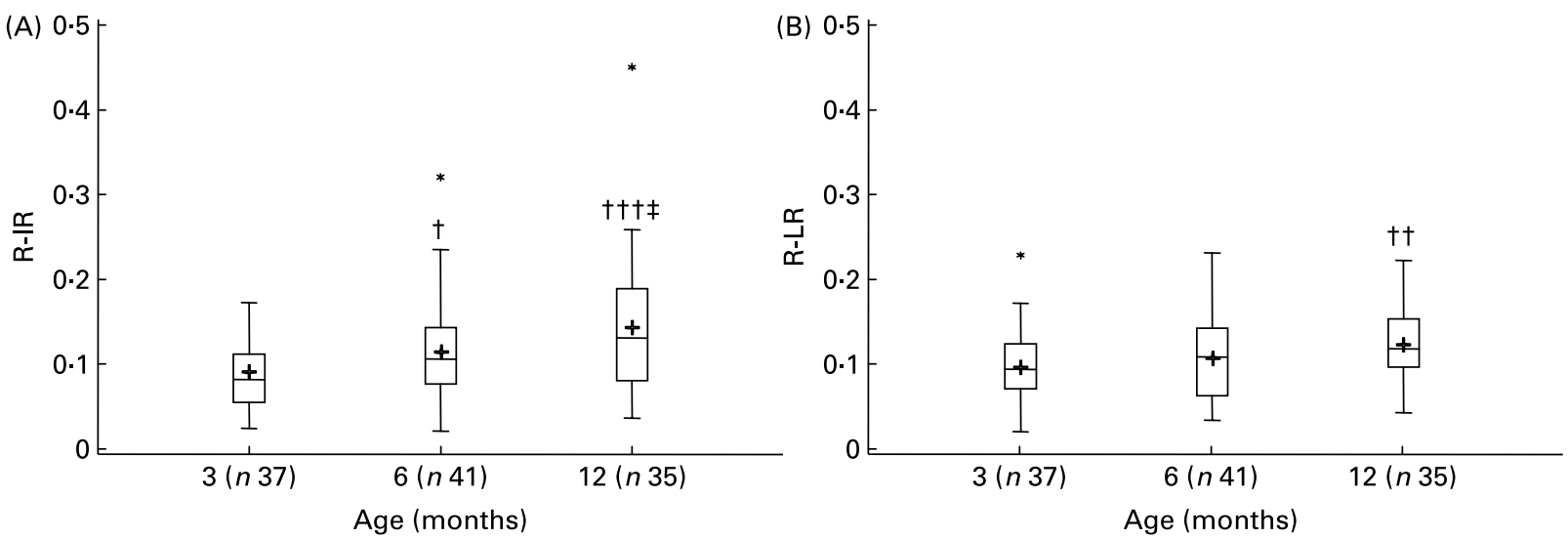

Fig. 2. Box plots of global taste reactivity based on ingestion ratios (R-IR) $(A)$ and global taste reactivity based on liking ratios (R-LR) (B) for each studied age (3, 6 and 12 months old). For each box plot, the bottom and the top of the box are the 25th and 75th percentiles and the line within the box is the median; the + sign is the mean. The whiskers extend from the box as far as the data extend, to a distance of at most $1.5 \times$ interquartile range. Any values more extreme than this are marked by $a *$. Mean value was significantly different from that at 3 months: $\dagger P=0.095$ (marginal), $\dagger+P=0.015, \dagger \dagger \dagger P=0.001$ (LSMEANS and $t$ tests). $\ddagger$ Mean value was marginally significantly different from that at 6 months $(P=0.069)$ (LSMEANS and $t$ tests). 
Table 2. Kendall correlations between ingestion ratio and liking ratio for each taste at each age studied

\begin{tabular}{|c|c|c|c|c|c|c|}
\hline & \multicolumn{2}{|c|}{3 months old } & \multicolumn{2}{|c|}{6 months old } & \multicolumn{2}{|c|}{12 months old } \\
\hline & Kendall's $\tau$ & $P$ & Kendall's $\tau$ & $P$ & Kendall's $\tau$ & $P$ \\
\hline Sweet taste & 0.23 & 0.0571 & 0.19 & 0.0958 & 0.54 & $<0.0001$ \\
\hline Salty taste & -0.07 & 0.5853 & 0.14 & 0.2233 & 0.48 & $<0.0001$ \\
\hline Bitter taste & 0.20 & 0.0986 & 0.24 & 0.0399 & 0.46 & 0.0002 \\
\hline Sour taste & 0.33 & 0.0081 & $0 \cdot 16$ & 0.1461 & 0.42 & 0.0007 \\
\hline Umami taste & 0.03 & 0.7885 & 0.41 & 0.0005 & 0.18 & 0.1757 \\
\hline
\end{tabular}

Not surprisingly, during the first year of life sweet taste was on average preferred over water. Moreover, the acceptance of sweet taste slightly decreased between 6 and 12 months old: it shifted from preference to indifference. These findings are consistent with previous studies conducted with sucrose used as a sweet stimulus: sweet taste acceptance remained stable from the ages of $1-3 \mathrm{~d}$ to $20-28$ weeks $^{(17)}$ and decreased between the ages of 4 and 6.7 months ${ }^{(20)}$. This decrease might be a continuous process until adulthood: preference for sweet taste has indeed been shown to decline between the age of $11-15$ years and the age of 19-25 years in a longitudinal study ${ }^{(31)}$. Several hypotheses were discussed to explain this developmental change, putting forward the role of physiological and experiential factors. During the first year of life one of the plausible explanations to this developmental change might be the effect of experience. The preference for sweet taste over water was indeed shown to be maintained in infants fed sweetened water during the first 6 months of life $\mathrm{e}^{(30,32)}$. Moreover, during the second half of the first year of life, the progressive replacement of milk, which tastes sweet, by solid foods, not all of which taste sweet, leads to a globally less sweet diet, which might drive the decrease in sweet taste acceptance. The decrease of sweet acceptance from 6 to 12 months could also be related to the food context of sweet experiences. With increasing age infants have more and more experiences with sweet-tasting foods (i.e. fruit purées, juices) and they most probably learn which food tastes sweet. Water could be a medium that they do not associate with sweetness.

Results on salty taste revealed that the preference for salty taste emerges between the ages of 3 and 6 months. This is in line with previous findings obtained using a more concentrated saline solution ${ }^{(20,21)}$. This phenomenon could be due to a maturation of the mechanisms underlying salt taste perception, such as a maturation of the receptors as reported in animals ${ }^{(20)}$, although no evidence of such a phenomenon is available for humans. Since some authors report either a neutral reaction $^{(33)}$ or a rejection of salty taste in newborns ${ }^{(18)}$, the validity of this hypothesis is questionable. The emergence of the preference for salt between the ages of 3 and 6 months might be due to an exposure effect, since this is the period by which a majority of infants are weaned and therefore potentially exposed to salty foods. Infants aged 6 months old were indeed shown to develop a preference for salt, linked to their recent dietary experience of $\mathrm{Na}$ in foods ${ }^{(34)}$. Between the ages of 2 and 12 months, $\mathrm{Na}$ intake increases as the diet changes over this period ${ }^{(35)}$, and intake at 12 months can vary by a factor of 2 depending on the given foods (i.e. baby foods or table foods) ${ }^{(36)}$. A survey, recently conducted in Dijon, showed that about a quarter of participating mothers added salt to weaning foods ${ }^{(37)}$.

Results based on ingestion revealed that infants were indifferent to bitter taste $(0 \cdot 18 \mathrm{M}$-urea) whereas results based on liking revealed a clear rejection of bitter taste. The indifference to bitter taste based on ingestion might seem puzzling in the sense that bitterness is generally considered to be rejected. However, this finding is in accordance with previous studies conducted at birth. Newborns display negative facial expressions when tasting a highly concentrated solution of quinine or urea ${ }^{(13,14)}$ but they do not modify their ingestion behaviour when tasting a solution of urea $(0.24$ and $0.48 \mathrm{M})$ in comparison with water or when tasting a solution of sucrose plus urea $(0.07 \mathrm{M}$-sucrose plus $0.18,0.24$ or $0.48 \mathrm{M}$-urea $)$ in comparison with the diluent ${ }^{(12)}$. A decrease in the consumption of a solution of sucrose plus urea was observed later at the age of 6 months ${ }^{(22)}$, and in a group of 2- to 24-monthold infants ${ }^{(23)}$. The discrepancy between those results and the present ones might be related to the medium used (sweet water $v$. water). It might also be due to the large age range of the infants ${ }^{(23)}$. The present study concludes that there is stability in urea acceptance between the ages of 3 and 12 months. This result does not agree with the findings of Kajiura et al. that showed a decrease between birth and the age of 6 months in urea acceptance measured through ingestion and hedonic ratings ${ }^{(22)}$. This discrepancy could be related to the appearance of a developmental change in urea acceptance between birth and 3 months of age. Besides, to explain the differences between studies in which quinine or urea were used, it must be remembered that there is a large number of bitter-tasting compounds and of bitter taste receptors (about twenty-five taste TAS2 receptors) ${ }^{(38)}$. As a consequence, the perception of a bitter-tasting compound would certainly not predict that of another bitter-tasting compound $^{(39)}$, which makes comparisons between studies using different bitter compounds difficult. In other respects, it is not possible to completely rule out the possibility that some infants did not perceive the bitter compound. However, since some infants displayed negative mimics when tasting the bitter solution and because the concentration used was supra-threshold, it seems unlikely that overall the infants did not perceive bitter taste. Yet, our findings clarify previous results: data relying on the experimenter's judgement, which was based both on ingestion and on the infant's facial expressions, indicated rejection on average at the three ages tested whereas the measure of ingestion did not systematically indicate a rejection. Finally, prenatal exposure to urea has to 
be considered to interpret urea taste acceptance. An early exposure due to urea in amniotic fluid (8.9 (SD 2.9) $\mathrm{mm}$ in fetal urine ${ }^{(40)}$ ) might be associated with 'tolerance' to this taste at the beginning of life.

Concerning sour taste, the liking measure indicated its rejection, whereas ingestion revealed indifference. Previous studies based on facial expressions also concluded that there is a rejection of sour stimuli by newborns ${ }^{(13,14,16)}$, and those based on ingestion concluded that there is a rejection of sour taste (presented in a mildly sweet solution) in newborns $^{(12)}$ and in 2- to 24-month-old infants ${ }^{(23)}$. On the contrary, no decrease in ingestion was evidenced when citric acid was presented in water in newborns ${ }^{(12)}$. The fact that the sour stimulus was added to a sweet solution in some studies could explain the discrepancy between their results and the present ones. Indeed, the contrast of preference between a sour solution over water and between a sweet and sour solution over sweet water might not be equivalent due to the positive value of sweet water and the neutral value of water $^{(12)}$. One might also wonder if infants could perceive the sour taste at the concentration used $(0 \cdot 006 \mathrm{M})$. The hypothesis of no detection of the sour taste is unlikely, since a majority of infants displayed negative facial expressions when tasting the sour solution, which contributed to the more negative judgement of liking expressed by the experimenter. However, other infants seemed to like the sour taste and to modify their ingestion accordingly: between 43 and $48 \%$ of individual IR were greater than 0.5 at all the ages tested. Interestingly, recent findings suggest that $23 \%$ of 15- to 20-month-old infants ${ }^{(24)}$ and $35 \%$ of 5- to 9-yearold children display a preference for very sour stimuli ${ }^{(25)}$. Because our approach was different (only one concentration was used here) we could not perform the same segmentation in sour taste acceptance. In other respects, the heightened sour preferences in infancy is associated with a higher consumption of fruit ${ }^{(24,41)}$ but it remains unknown whether exposure to fruits, some of which taste sour, was the driver of a higher sour acceptance or if this heightened sour preference was already present at birth. Since we observed a marginal increase in inter-individual variability in ingestion of sour solutions over the first year, the effect of exposure seems plausible but remains to be demonstrated. Moreover, a predisposition to prefer sour stimuli at birth in some infants cannot be ruled out. A genetic influence on sour taste perception has not been evidenced yet contrarily to bitter taste perception $^{(42)}$.

The acceptance of umami taste has received little attention in the past. In newborns, Steiner described the same facial mimics after the presentation of an umami-tasting soup as the ones displayed after tasting a sweet stimulus ${ }^{(26)}$ which was interpreted as a preference for umami taste. Another study showed that 2-month-old infants rejected a solution of MSG over water whereas 7-month-old infants were overall indifferent to $\mathrm{it}^{(43)}$. The acceptance of umami taste probably depends on the vehicle: in 2- to 24-month-old infants the addition of MSG to water reduced its ingestion ${ }^{(27)}$ whereas the addition of MSG to a vegetable soup increases its ingestion $^{(23)}$. This last observation combined with the present observation of no clear rejection of MSG in water in 3-, 6and 12-month-old infants can lead to the hypothesis of a decrease in the umami taste acceptance in water occurring after the age of 12 months. This does not exclude the possibility that MSG was not detected at the concentration used $(0.009 \mathrm{M})$, especially at the age of 3 months. However, this hypothesis seems unlikely since at this concentration, MSG is above its detection threshold in adults ${ }^{(44)}$. Moreover, the fact that we observed a large inter-individual variability in the umami taste acceptance does not reinforce the hypothesis of a systematic lack of detection of umami.

The fact that evolutions were not uniform across tastes over the first year (i.e. changes in acceptance did not occur for bitter, sour and umami tastes and occurred differently for sweet and salty tastes) confirms that these evolutions are not due to a general change in taste perception. Moreover, the inter-individual differences in taste acceptance globally increased over the first year. The possible effects of experience cannot be turned down. Nevertheless, it does not exclude a specific developmental change for some tastes such as salty taste, for example. The weight of exposure effects among other determinants such as physiological factors (a potential maturation of receptors, for example) remains unknown and has to be demonstrated.

An original finding of the present study was that the ingestion behaviour (i.e. IR) and the infant's global behaviour estimated by the experimenter's judgment of the infant's liking (i.e. LR) were two complementary measures to assess taste acceptance in infants. This seemed to be particularly true for 'disliked' tastes such as bitter and sour tastes. A grimace was not systematically associated with a reduced ingestion. The same observation was carried out in a group of ten 6-month-old infants ${ }^{(45)}$ whose taste acceptance was studied with the same procedure (data from five out of these ten infants were included in the present paper) but for which facial mimics were analysed with the objective Baby FACS: Facial Action Coding System for Infants and Young Children $₫ \operatorname{method}^{(46)}$. The separate neural mechanisms underlying wanting (as measured by ingestion) and liking (as measured by the experimenter's judgment of the infant's liking $)^{(47,48)}$ and their putative different developmental patterns could explain the discrepancy between the conclusions from both measures. This hypothesis could account for previous conflicting results on the development of taste acceptance, and is supported by Doty \& Shah's ${ }^{(49)}$ discussion (p. 306): 'In general, behavioural responses to tastants, particularly unpleasant ones, are initially reflex-like in nature but become more voluntary over the course of the verbal first year of life. [...] Thus, spontaneous facial expressions to taste stimuli, which are fairly stable over the first postnatal months, gradually decrease, being replaced by more noticeable intentional behaviours such as refusal to open mouth or pushing the spoon away with the hands.' In our view the features of the facial mimics might be relatively stable over the first year but their intensities might increase. Thus, their meaning for caregivers might evolve as the infant learns to use these mimics to communicate ${ }^{(50)}$. At the same time, motor and cognitive development allows the infant to control more and more his/her head and body language. As a consequence, the congruency between ingestion and liking significantly increased during the first year.

Whatever the measure considered, the average global taste reactivity (i.e. R-IR and R-LR) increased over the first year. In other words, the reactions to basic tastes were more and more contrasted with age. The effects of exposure might 
partly explain this phenomenon since the infant's diet changes from a milk homogeneous diet to a diversified diet over the first year.

Although the psycho-affective aspect of eating cannot be ignored in the development of food behaviour, especially in infants and children, this aspect is often neglected in research. By contributing to a better understanding of the development of taste acceptance over the first year the present study enhances the comprehension of food behaviour in infancy, the foundation of food behaviour in adulthood. It confirmed in particular the early attraction to sweet and salty tastes, which might later drive the appetite for sweet and salty foods. Given the large availability of such foods targeted to children, it is especially important to understand the role of taste preferences on food acceptance in this population in a context of increasing childhood obesity. The present study represents a first step towards this comprehension. Future studies linking taste acceptance and food preferences in infancy should follow on to fully explore the early formation of food behaviour.

\section{Acknowledgements}

The present study was carried out with the financial support of the Regional Council of Burgundy, IFR92, PRNH-INRAINSERM and the Agence Nationale de la Recherche (ANR; The French National Research Agency) under the 'Programme National de Recherche en Alimentation et Nutrition Humaine' (project ANR-06-PNRA-028, OPALINE). The present study was supported by grants from Blédina, Nestlé, Symrise and CEDUS; branded as Vitagora ${ }^{\circledR}$ (The Taste, Nutrition and Health Innovation Pole). C. S. wishes to thank the Benjamin Delessert Institute for the 'Prix de Recherche' 2008. The authors wish to thank the infants and parents who took part in the present study, and the experimenters: E. Szleper, V. Feyen, J. Pierard and F. Durey for their technical support, C. Laval for the recruitment, C. Chabanet for her statistical advice, V. Boggio for his advice and the OPALINE team for its general support.

C. S. and S. N. designed the study with the help of S. I. The collection of the data, the data analysis and the writing of the paper were performed by C. S. with the help of S. I. and S. N.

None of the authors has any conflict of interest to report.

\section{References}

1. Birch LL (1979) Preschool children's food preferences and consumption patterns. J Nutr Educ 11, 189-192.

2. Capaldi ED \& Privitera GJ (2008) Decreasing dislike for sour and bitter in children and adults. Appetite 50, 139-145.

3. Gibson EL, Wardle J \& Watts CJ (1998) Fruit and vegetable consumption, nutritional knowledge and beliefs in mothers and children. Appetite 31, 205-228.

4. Havermans RC \& Jansen A (2007) Increasing children's liking of vegetables through flavour-flavour learning. Appetite $\mathbf{4 8}$, 259-262.

5. Nicklaus S, Chabanet C, Boggio V, et al. (2005) Food choices at lunch during the third year of life: increase in energy intake but decrease in variety. Acta Paediatr 94, 1023-1029.

6. Nicklaus S, Boggio V \& Issanchou S (2005) Food choices at lunch during the third year of life: high selection of animal and starchy foods but avoidance of vegetables. Acta Paediatr 94, 943-951.

7. Skinner JD, Carruth BR, Bounds W, et al. (2002) Children's food preferences: a longitudinal analysis. J Am Diet Assoc 102, $1638-1647$.

8. Nicklaus S, Boggio V, Chabanet C, et al. (2005) A prospective study of food variety seeking in childhood, adolescence and early adult life. Appetite 44, 289-297.

9. Nicklaus S, Boggio V, Chabanet C, et al. (2004) A prospective study of food preferences in childhood. Food Qual Pref 15, $805-818$.

10. Nicklas TA, Webber LS \& Berenson GS (1991) Studies of consistency of dietary intake during the first four years of life in a prospective analysis: Bogalusa Heart Study. J Am Coll Nutr 10, 234-241.

11. Devine CM, Connors M, Bisogni CA, et al. (1998) Life-course influences on fruit and vegetable trajectories: qualitative analysis of food choices. J Nutr Educ 30, 361-370.

12. Desor JA, Maller O \& Andrews K (1975) Ingestive responses of human newborns to salty, sour, and bitter stimuli. J Comp Physiol Psychol 89, 966-970.

13. Steiner JE (1979) Human facial expressions in response to taste and smell stimulation. Adv Child Dev Behav 13, 257-295.

14. Ganchrow JR, Steiner JE \& Daher M (1983) Neonatal facial expressions in response to different qualities and intensities of gustatory stimuli. Infant Behav Dev 6, 473-484.

15. Ganchrow JR \& Mennella JA (2003) The ontogeny of human flavor perception. In Handbook of Olfaction and Gustation, 2nd ed., pp. 823-846 [RL Doty, editor]. New York: Marcel Dekker.

16. Rosenstein D \& Oster H (1988) Differential facial responses to four basic tastes in newborns. Child Dev 59, 1555-1568.

17. Desor JA, Maller O \& Turner RE (1977) Preference for sweet in humans: infants, children and adults. In Taste and Development: The Genesis of Sweet Preference, pp. 161-172 [JM Weiffenbach, editor]. Washington, DC: US Government Printing Office.

18. Crook CK (1978) Taste perception in the newborn infant. Infant Behav Dev 1, 52-69.

19. Desor JA, Maller O \& Turner RE (1973) Taste in acceptance of sugars by human infants. J Comp Physiol Psychol 84, 496-501.

20. Beauchamp GK, Cowart BJ \& Moran M (1986) Developmental changes in salt acceptability in human infants. Dev Psychobiol 19, $17-25$.

21. Beauchamp GK, Cowart BJ, Mennella JA, et al. (1994) Infant salt taste: developmental, methodogical, and contextual factors. Dev Psychobiol 27, 353-365.

22. Kajiura H, Cowart BJ \& Beauchamp GK (1992) Early developmental change in bitter taste responses in human infants. Dev Psychobiol 25, 375-386.

23. Vasquez M, Pearson PB \& Beauchamp GK (1982) Flavor preferences in malnourished Mexican infants. Physiol Behav 28, 513-519.

24. Blossfeld I, Collins A, Boland S, et al. (2007) Relationships between acceptance of sour taste and fruit intakes in 18-month-old infants. Br J Nutr 98, 1084-1091.

25. Liem DG \& Mennella JA (2003) Heightened sour preferences during childhood. Chem Senses 28, 173-180.

26. Steiner JE (1987) What the neonate can tell us about umami. In Umami: A Basic Taste, pp. 97-123 [Y Kawamura and MR Kare, editors]. New York: Marcel Dekker.

27. Beauchamp GK \& Pearson P (1991) Human development and umami taste. Physiol Behav 49, 1009-1012.

28. Cowart BJ (1981) Development of taste perception in humans: sensitivity and preference throughout the life span. Psychol Bull 90, 43-73.

29. Jensen RG (1995) Handbook of Milk Composition. New York: Academic Press. 
30. Beauchamp GK \& Moran M (1982) Dietary experience and sweet taste preferences in human infants. Appetite 3, 139-152.

31. Desor JA \& Beauchamp GK (1987) Longitudinal changes in sweet preferences in humans. Physiol Behav 39, 639-641.

32. Beauchamp GK \& Moran M (1984) Acceptance of sweet and salty tastes in 2-year-old children. Appetite 5, 291-305.

33. Maller O \& Desor JA (1973) Effect of taste on ingestion by human newborns. In Fourth Symposium on Oral Sensation and Perception, pp. 279-291 [JF Bosma, editor]. Bethesda, MD: US Department of Health, Education and Welfare.

34. Harris G \& Booth DA (1987) Infants' preference for salt in food: its dependence upon recent dietary experience. $J$ Reprod Infant Psychol 5, 97-104.

35. Fantino M \& Gourmet E (2008) Apports nutritionnels en France en 2005 chez les enfants non allaités âgés de moins de 36 mois (Nutrient intakes in 2005 by non-breast fed French children of less than 36 months). Arch Pediatr 15, 446-455.

36. Mosser F (2005) Le sel: vigilance dans la première année de vie (Salt: vigilance in the first year of life). Cah Nutr Diet 40 , 202-206.

37. Maier A, Chabanet C, Schaal B, et al. (2007) Food-related sensory experience from birth through weaning: contrasted patterns in two nearby European regions. Appetite 49, 429-440.

38. Behrens M, Foerster S, Staehler F, et al. (2007) Gustatory expression pattern of the human TAS2R bitter receptor gene family reveals a heterogenous population of bitter responsive taste receptor cells. J Neurosci 27, 12630-12640.

39. Delwiche FJ, Buletic Z \& Breslin P (2001) Covariation in individuals' sensitivities to bitter compounds: evidence supporting multiple receptor/transduction mechanisms. Percept Psychophys 63, 761-776.
40. Muller F, Dommergues M, Bussières L, et al. (1996) Development of human renal function: reference intervals for 10 biochemical markers in fetal urine. Clin Chem 42, 1855-1860.

41. Liem DG, Bogers RP, Dagnelie PC, et al. (2006) Fruit consumption of boys (8-11 years) is related to preferences for sour taste. Appetite 46, 93-96.

42. Mennella JA, Pepino MY \& Reed DR (2005) Genetic and environmental determinants of bitter perception and sweet preferences. Pediatrics 115, e216-e222.

43. Beauchamp GK, Bachmanov A \& Stein LJ (1998) Development and genetics of glutamate taste preference. Ann N Y Acad Sci $\mathbf{8 5 5}, 412-416$.

44. Lugaz O, Pillias AM \& Faurion A (2002) A new specific ageusia: some humans cannot taste L-glutamate. Chem Senses 27, $105-115$.

45. Schwartz C, Chabanet C, Issanchou S, et al. (2008) Reactivity to basic tastes in 6-month-old infants: comparison of intake and Baby FACS data. In 12th European Conference on Facial Expression. Geneva: NCCR in Affective Sciences.

46. Oster H (2007) Baby FACS: Facial Action Coding System for Infants and Young Children $(\mathcal{C}$. New York University.

47. Berridge $\mathrm{KC}(2000)$ Measuring hedonic impact in animals and infants: microstructure of affective taste reactivity patterns. Neurosci Biobehav Rev 24, 173-198.

48. Berridge KC (1996) Food reward: brain substrates of wanting and liking. Neurosci Biobehav Rev 20, 1-25.

49. Doty RL \& Shah M (2008) Taste and smell. In Encyclopedia of Infant and Early Childhood Development, pp. 299-308 [MH Marshall and BB Janette, editors]. Oxford: Academic Press.

50. Chiva M (1985) Le Doux et L'amer. Croissance de L'enfant Genèse de L'homme (Sweet and Bitter. Child Growth Genesis of Man). Paris: PUF. 